\title{
FEATURE BASED OPINION CLASSIFICATION (FBOC) OF CUSTOMER REVIEWS
}

\author{
Ankur Ratmele \\ Research Scholar, IET DAVV Indore (M.P.), India \\ Assistant Professor, NMIMS Indore (M.P.), India \\ ankur22ratmele@gmail.com \\ Ramesh Thakur \\ Associate Professor, IIPS, DAVV, Indore (M.P.), India \\ r_thakur@rediffmail.com
}

\begin{abstract}
In the era of Web 2.0 e-commerce website plays a very important role in our everyday life. If the consumer wants to purchase a product from the e-commerce website then reviews plays a very important role in decision making about a product. The reviews are unstructured reviews because there is no fixed format for customer to write the reviews. Because of these huge quantities of unstructured reviews there is a need to develop a technique which extract the features from the reviews and gives the opinion about the features. In this proposed technique i.e Features Based Opinion Classification (FBOC), identification of features is done, then after the identification, feature is classified as according to their opinion whether positive, negative and neutral using two methods namely AFINN and VADER (Valence Aware Dictionary for sEntiment Reasoning). Summary of feature is generated showing total number of positive, negative and neutral opinion of features and visualized in a form such that it can be easily understandable by end user.
\end{abstract}

Keywords: FBOC, AFINN, VADER, FE(Feature Extraction),

\section{Introduction}

With Web 2.0's rising popularity, opinion mining is becoming ever more exciting. New forms of opinion rich outlets emerged such as forums, social networks, wikis and websites reviews, e-commerce etc. We need to self regulate their extraction and organization, due to the biggest size of such available data. In earlier days when the buyer wants to buy the product then buyer has to ask people regarding the product and its features. The buyer needs to find the people who had the product and then the buyer needs to ask a lot of people about the product and its features. Features play a very important role in order to buy product because people are interested to know which features are good for this product. But with the introduction of web 2.0 online shopping is very popular in reality, customers can write reviews about the product's feature and buyers who want to purchase the product can read those reviews and decide whether to purchase it or not.

Following are some important terms in opinion mining they are:

i. Feature:

A feature is an aspect of the product that has been commented on in reviews.

ii. Opinion:

The opinion of a feature in reviews is clause that conveys an opinion on the feature. Opinion can be positive opinion, negative opinion and neutral opinion (Pang and Lee, 2008). Positive opinion means customer mentioned positive attitude towards the feature of product. Negative opinion means customer mentioned negative attitude towards the feature of product. Neutral opinion means customer mentioned neither positive nor negative attitude towards the feature of product.

In this FBOC there are two challenging task

a. Identify the features from the reviews

b. For identified feature find their opinion based on reviews.

In this proposed technique we have downloaded reviews from amazon website. We applied LDA in order to identify the features from the reviews and after the identification of feature; opinion of the identified feature is extracted using two lexicon based methods AFINN and VADER.

In the remainder of this paper, we would like to share related work in next section, after that we will discuss our proposed technique, experiment and results followed by conclusion. 


\section{Related Work}

(Homoceanu et al., 2011), developed a technique for automatically analyzing user reviews. The results of this investigation are then used for grouping of reviews based on semantics. By applying some probabilistic approach they have extracted features.

(Sun et al., 2014) recommended a method of implicit feature extraction based on context. They pull out the implicit features according to the word of opinion and the likeness between the features of the product. They create a matrix to illustrate the association between the words of opinion and the features of the product, then they filter the noises in the content. They use ictclas $4 \mathrm{j}$ word segmentation tools to segment the comments and also use similarity score to identify implicit features. This method has certain flaws, e.g. in the small-scale corpora, it can't well understand the implicit features.

(Hu and Liu , 2004) proposed a technique that extracts frequent nouns as features of the products. Product features set in their paper are established by using Apriori algorithm to pull out frequent words and remove some common words. However, the method that they have proposed is concerned only with product features that people habitually use, while the rare terms that may be correct product features are sometimes ignored or may be more important than words that are commonly used.

(Pang and Lee, 2008) utilized a SentiWordNet based scheme with two linguistic feature selections such as adjectives, adverbs, verbs, and extraction of n-gram features.

(Aires et al., 2018) used deep learning methods to know sentiment at aspect-level. They used deep learning approaches for both recognition and classing of aspects and a preprocessing phase that uplift the results for aspect recognition.

(Bouchlaghem et al., 2010) proposed a technique for extracting and classifying opinions on the basis of semantic similarities between words. Their technique consists of three stages for classifying the opinion in a text passage, starting with the pre-processing process which consists of splitting of text and tagging its words. P-SIM based algorithm allows for the extraction of opinion-oriented terms in a second step. Finally, with respect to the words derived, the polarity of general opinion is predicted. In order to make a decision which words are opinionoriented, they proposed an algorithm which assigns a score to each word allowing its polarity to be determined.

(Zhixing Li, 2015) proposed a technique which combines the methodology of associative classification with the overall rating to determine the relationship between opinion and feature terms.

(Xu et al., 2010) introduced a Chinese product features identification approach based on a Conditional Random Fields model on combining chunk features and heuristic location knowledge in accumulation to term features, part-of speech features and background features.

(Song et al., 2011) proposed a technique for the automated retrieval of title and opinion terms from customerpublished comments. In this extraction of POS patterns in according to the range, frequency, upper likelihood and lower pattern limit, and some patterns were merged into the fuzzy form.

(Sun et al., 2009), proposed a system for comparing different products practicing customer recommendations and visualizing the results and also people can judge against the products on feature level. In fact, through contrast the consumer can clearly say the strengths and disadvantages of each item.

Some research works (Wu et al., 2009; Yan et al., 2015; V. Ravi Kumar et al., 2012; Qadir .A, 2009; Hutto and Gilbert, 2014; Nielsen F, 2011) recognized these features using relationship-dependence based on relationships between words that appeared in reviews.

(Chen et al., 2019), proposed a way to make the most the linguistic features of negative and extensive words in conjunction with the context facts and additionally this, technique solve domain specific problem.

(Ito et al. ,2020), proposed a contextual sentiment neural network (CSNN) and a novel learning strategy called initialization and propagation (IP) learning, they demonstrated the effectiveness of IP learning for improving the explainability of CSNN.

(Ratmele et al., 2015) survey shows the various data sets and techniques that are available in the area of opinion mining and sentiment analysis. 


\section{Proposed Technique}

The overall architecture of the proposed technique is shown in Fig. 1. In this proposed technique the dataset taken from the amazon e-commerce website [16]. Following are the various steps used in the proposed technique:

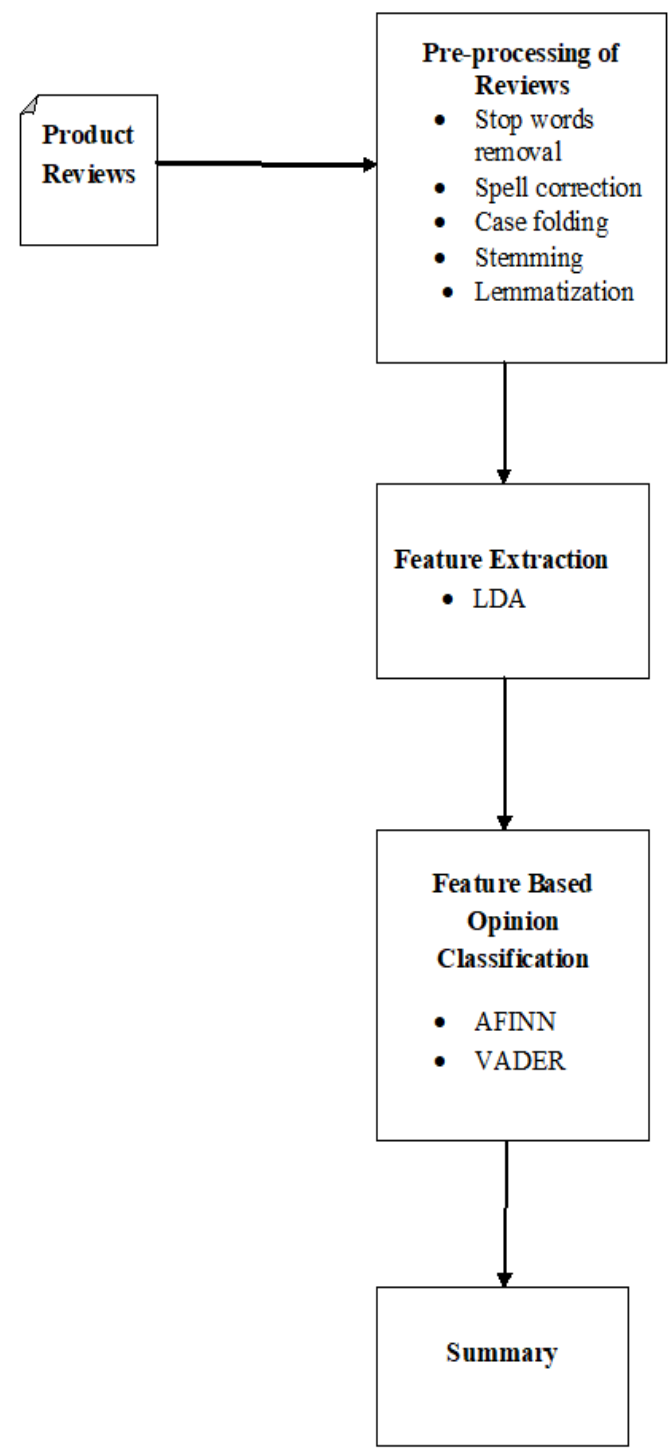

Fig. 1. Proposed architecture of FBOC

\subsection{Product Reviews}

In this technique reviews of mobile phone is used which is extracted from amazon e-commerce website and stored in csv file. Total number of reviews is 2760 which is of free format containing positive, negative and neutral opinion of the mobile phone.

\subsection{Preprocessing of reviews}

Reviews that are stored in csv file is of free format so preprocessing has to be done. The reviews contain lots of stop words which have to be removed because it is of no use. Spelling correction is required in order to correct the spelling in reviews. Next is Case folding, which means converting text into lower case because the reviews are the collection of upper and lower case. Stemming and Lemmatization needs to be done in order to make data ready for further processing.

\subsection{Feature Extraction}

Previously many researchers have identified a feature manually or with the use of Part of Speech Tagging, in which noun is identified as features of the product but in our technique we have used Latent Dirichlet Allocation (LDA) to identify the features. Algorithm of feature extraction in FBOC is shown below: 


\section{Algorithm of Feature Extraction (FE)}

Input: $\mathrm{RS}=\left\{\mathrm{R}_{1}, \mathrm{R}_{2}, \mathrm{R}_{3} \ldots \ldots \ldots \ldots \mathrm{R}_{\mathrm{n}}\right\}$

$\mathrm{RS}=$ Set of Reviews.

Output: $F S=\left\{F_{1}, F 2, F_{3}\right.$

$\mathrm{FS}=$ Set of Features

FE(RS)

1. begin

2. for each $R_{i}$ in $R S, i=1$ to $n$ do:

2.1 Casefold $R_{i}$ and store the result into $r_{i}, R_{i} \longrightarrow r_{i}$,

2.2 if $r_{i}$ contains stopwords then

$$
r_{i}^{\prime}=r_{i}-\{\text { stopwords }\}
$$

else

$$
r_{i}^{\prime}=r_{i} \quad / / \text { store } r_{i} \text { into } r_{i}^{\prime}
$$

2.3 perform Portstemmer and store the result into $r_{i}{ }^{\prime \prime}$ return $r_{i}^{\prime \prime}$

3. integrate $\mathrm{R}^{\prime}=\left\{\mathrm{r}_{1}{ }^{\prime \prime}, \mathrm{r}_{2}{ }^{\prime \prime}, \ldots . \mathrm{r}_{\mathrm{n}}{ }^{\prime \prime}\right\}$

4. pass $R^{\prime}$ into LDA to extract features

$\operatorname{LDA}\left(\mathrm{R}^{\prime}\right)$

return $\left(\mathrm{F}_{1}, \mathrm{~F}_{2}, \mathrm{~F}_{3} \ldots \ldots \ldots \ldots . \mathrm{F}_{\mathrm{n}}\right)$

5. integrate $\mathrm{FS}=\left\{\mathrm{F}_{1}, \mathrm{~F}_{2} \ldots \ldots \ldots \ldots \mathrm{F}_{\mathrm{n}}\right\}$

6. exit

With the help of above algorithm we are able to identify the features. From the identified features we have taken five features for Feature Based Opinion classification (FBOC). Identified features are Camera, Display, Battery, Performance and Money. The count of individual five features that we have used in this technique is shown in Fig. 2.

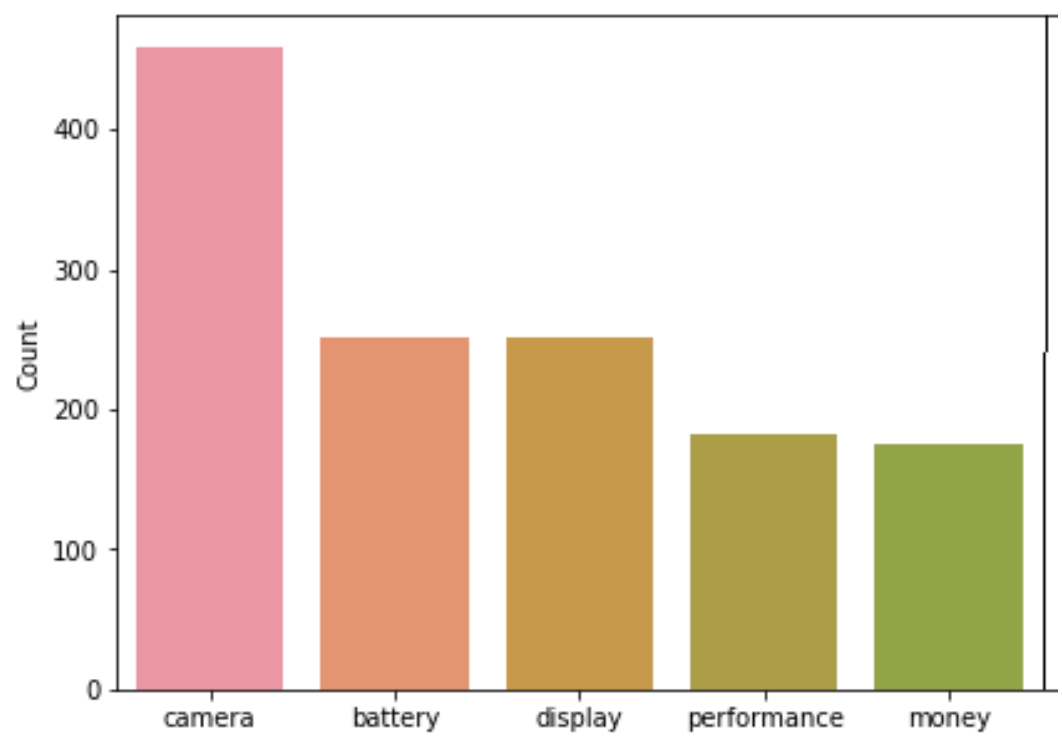

\subsection{Feature Based Opinion Classification (FBOC)}

Fig. 2. Frequency of identified Feature

We have extracted and identified five features of a mobile phone from above algorithm. After the identification of features we have applied lexicon based method AFINN and VADER to classify the features according to their opinion.

\subsection{Summary}

In this proposed technique, feature based summary of opinion is generated as shown in Table 1 and Table 2; containing total number of positive, negative and neutral opinion based on the feature using VADER and AFINN. 


\section{Experiment and Results}

In this work we have used lexicon based method AFINN and VADER to classify feature based on their opinion. The summary which indicates the total number of positive, negative and neutral opinion is shown in Table 1 and Table 2. Pr and A are Practical values and Actual values respectively shown in Table 1 and Table 2. Visual form of representation is shown in Fig. 3 and Fig. 5 which can be easily understandable by the user. Overall Opinion can be calculated by the following equation:

$$
\text { Overall Opinion }=\sum_{k}^{n} P \cup \sum_{k}^{n} N e \cup \sum_{k}^{n} N u
$$

where, $\mathrm{k}=1$ and $\mathrm{P}, \mathrm{Ne}$ and $\mathrm{Nu}$ are Positive Opinion, Negative Opinion and Neutral Opinion. So Overall Opinion is the union of all positive, negative and neutral opinion. Overall Opinion that is calculated by using Eq. (1) is shown in Fig. 4 and Fig. 6.

\subsection{VADER}

VADER (Hutto and Gilbert, 2014) is Valence Aware Dictionary for sEntiment Reasoning), a simple rule-based model for general sentiment analysis. By exposing both the lexicon and rule-based model, VADER makes the inner workings of the sentiment analysis engine more accessible to a broader human audience beyond the computer science community. VADER is capable of detecting polarity (positive, neutral, negative) of opinion in the reviews.

Table 1. Opinion Based Classification of Features using VADER

\begin{tabular}{|c|c|c|c|c|c|c|}
\hline \multirow{2}{*}{ Features } & \multicolumn{2}{|c|}{ \#Positive } & \multicolumn{2}{c|}{ \#Negative } & \multicolumn{2}{c|}{ \#Neutral } \\
\cline { 2 - 7 } & Pr & A & Pr & A & Pr & A \\
\hline Battery & 203 & 205 & 35 & 26 & 11 & 18 \\
\hline Camera & 397 & 397 & 18 & 15 & 14 & 17 \\
\hline Display & 225 & 225 & 15 & 15 & 9 & 9 \\
\hline Money & 157 & 157 & 13 & 10 & 2 & 5 \\
\hline Performance & 152 & 152 & 11 & 10 & 5 & 6 \\
\hline Overall Opinion & 1134 & 1136 & 92 & 76 & 41 & 55 \\
\hline
\end{tabular}

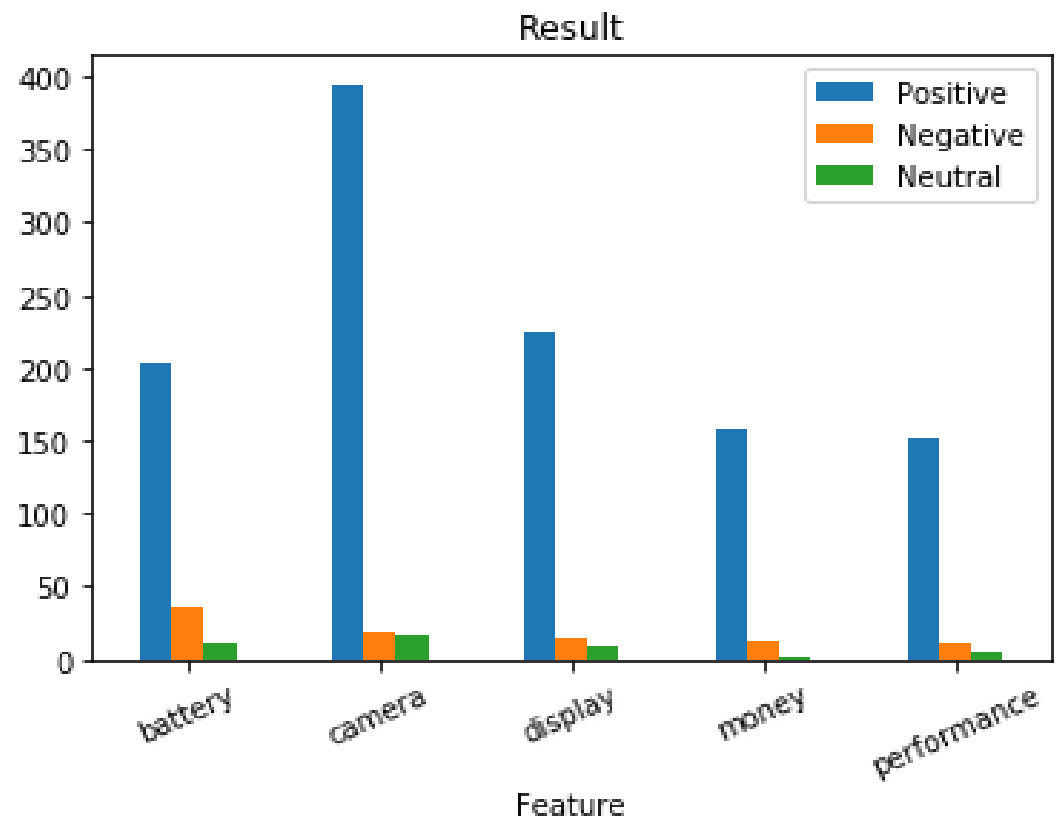

Fig. 3. Visualization of FBOC using VADER 


\section{Overall Opinion}

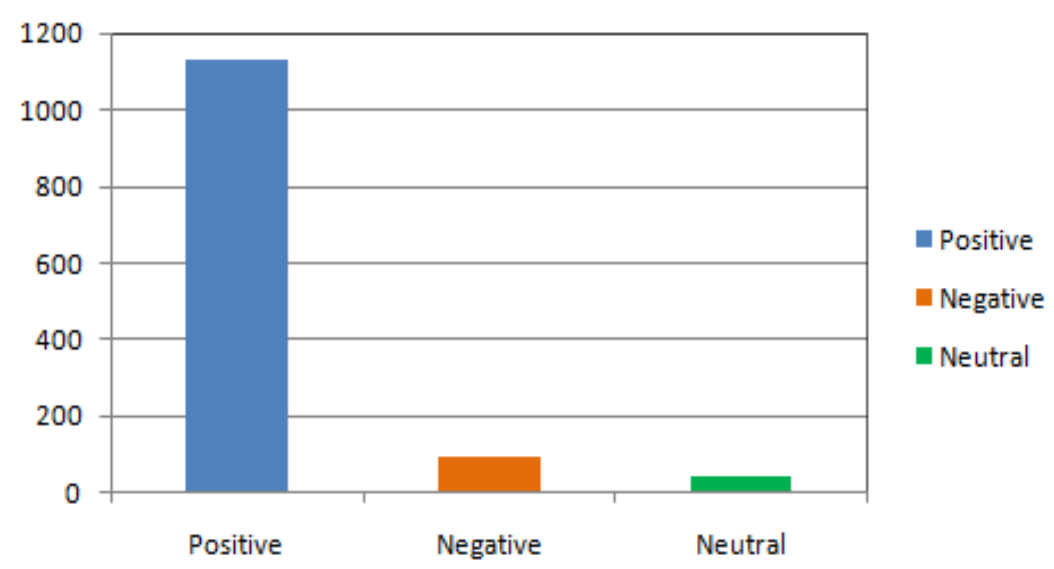

Fig. 4: Overall opinion of product using VADER

\subsection{AFINN}

The AFINN (Nielsen F , 2011) lexicon is a list of English terms manually rated for valence with an integer between -5 (negative) and +5 (positive) by Finn Årup Nielsen AFINN, review with a 0 sentiment score were classified as neutral, while those with scores greater 0 and lower than 0 were classified as positive and negative, respectively.

Table 2. Opinion Based Classification of Features using AFINN

\begin{tabular}{|c|c|c|c|c|c|c|}
\hline \multirow{2}{*}{ Features } & \multicolumn{2}{|c|}{ \#Positive } & \multicolumn{2}{c|}{ \#Negative } & \multicolumn{2}{c|}{ \#Neutral } \\
\cline { 2 - 7 } & Pr & A & Pr & A & Pr & A \\
\hline Battery & 203 & 205 & 23 & 26 & 23 & 18 \\
\hline Camera & 397 & 397 & 14 & 15 & 18 & 17 \\
\hline Display & 223 & 225 & 15 & 15 & 11 & 9 \\
\hline Money & 131 & 157 & 15 & 10 & 26 & 5 \\
\hline Performance & 149 & 152 & 10 & 10 & 9 & 6 \\
\hline Overall Opinion & 1103 & 1136 & 77 & 76 & 87 & 55 \\
\hline
\end{tabular}

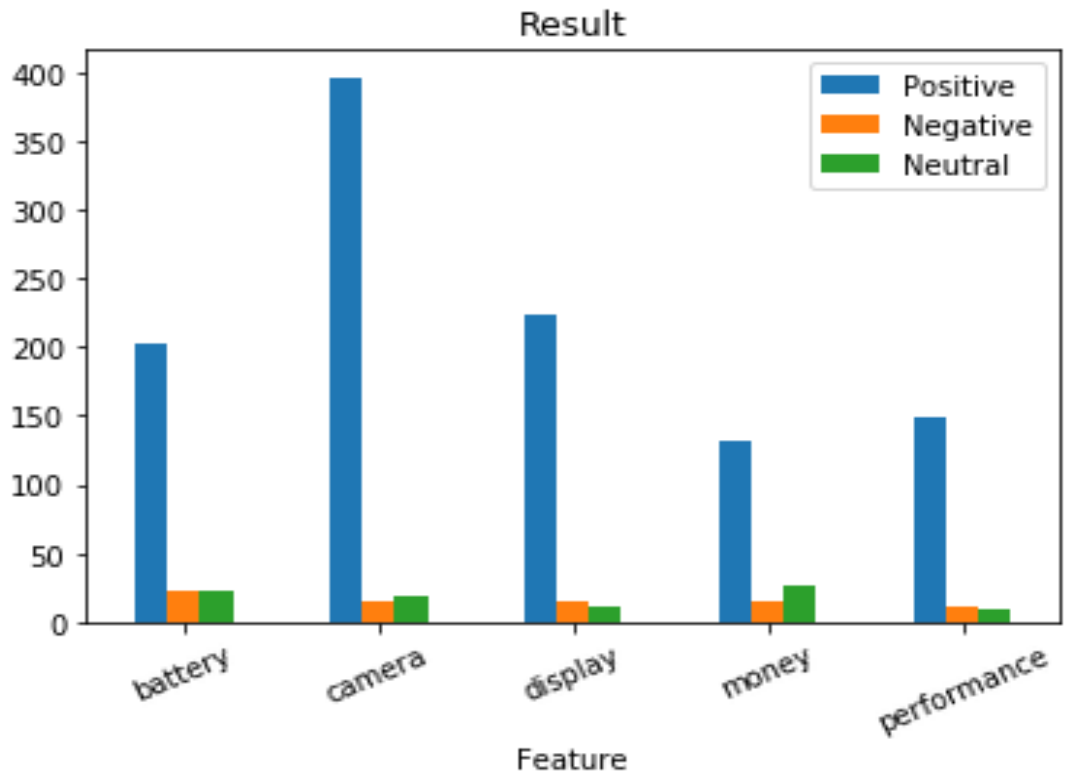

Fig. 5. Visualization of FBOC using AFINN 
overall opinion

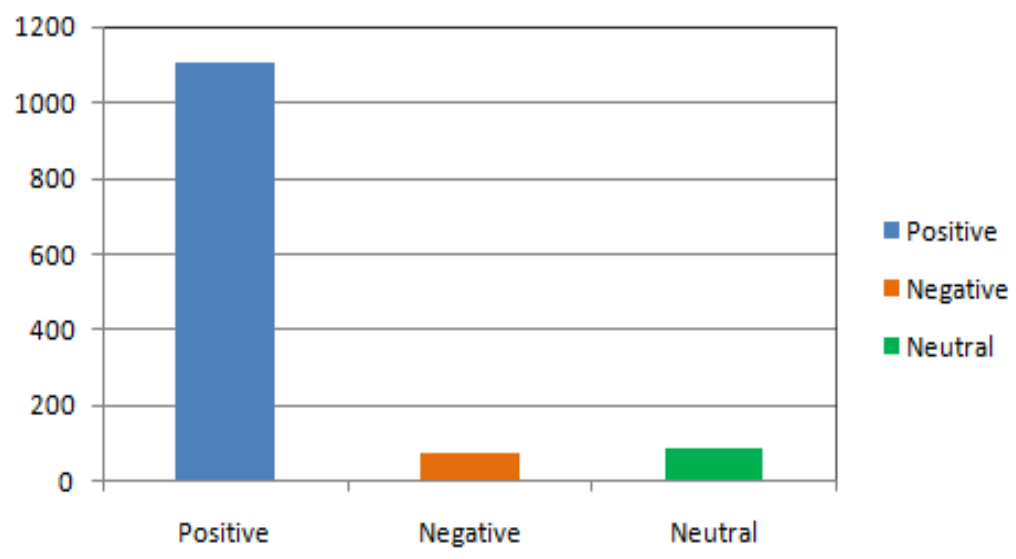

Fig. 6. Overall Opinion of product using AFINN

\section{Conclusion}

In this research work we have proposed a technique for Feature Extraction and opinion classification i.e Feature Based Opinion classification, in which features are extracted by using LDA and extracted features are then classified by lexicon based technique known as AFINN and VADER. From the results it is observed that VADER performs well on positive opinion while AFINN performs well on classifying Negative opinion. This work has done without POS tagging and it is effective in performing their task. If there are large numbers of product reviews exist then with the help of proposed technique this becomes much easier for users to know about the features and opinion of that feature.

\section{References:}

[1] Aires J. P., Padilha C., Quevedo C. and Meneguzzi F., "A Deep Learning Approach to Classify Aspect-Level Sentiment using Small Datasets," 2018 International Joint Conference on Neural Networks (IJCNN), Rio de Janeiro, 2018, pp. 1-8.

[2] Amazon Website https://www.amazon.in/Vivo-V11-Starry-Night-Offers/dp/B07HKK6K1X. Accesed 20 June 2020

[3] Bouchlaghem R., Elkhlifi A. and Faiz R., "Automatic extraction and classification approach of opinions in texts," 2010 10th International Conference on Intelligent Systems Design and Applications, Cairo, 2010, pp. 918-922.

[4] Chen, X., Rao, Y., Xie, H. et al. Sentiment Classification Using Negative and Intensive Sentiment Supplement Information. Data Sci. Eng. 4, 109-118 (2019)

[5] Homoceanu S., Loster M., Lofi C. and Balke W., "Will I Like It? Providing Product Overviews Based on Opinion Excerpts," 2011 IEEE 13th Conference on Commerce and Enterprise Computing, Luxembourg, 2011, pp. 26-33.

[6] Hu, M., and Liu, B., "Mining and summarizing customer reviews, " In Proceedings of the tenth ACM SIGKDD International Conference on Knowledge Discovery and Data Mining, Seattle,Wa, USA, pp. 342-351, 2004

[7] Hutto C, Gilbert E (2014) VADER: a parsimonious rule-based model for sentiment analysis of social media text. In: 8th international AAAI conference on weblogs and social media (ICWSM).

[8] Ito, T., Tsubouchi, K., Sakaji, H. et al. Contextual Sentiment Neural Network for Document Sentiment Analysis. Data Sci. Eng. 5, 180$192(2020)$.

[9] Nielsen F (2011) A new ANEW: evaluation of a word list for sentiment analysis in microblogs. arXiv:1103.2903

[10] Qadir, A.,"Detecting Opinion Sentences Specific to Product Features in Customer Reviews using Typed Dependency Relations," In: Events in Emerging Text Types (eETTs), Borovets, Bulgaria, 2009, pp. 38-43.

[11] Ratmele, Ankur \& Thakur, Ramesh. (2019). Statistical Analysis \& Survey of Research Work in Opinion Mining. SSRN Electronic Journal. 10.2139/ssrn.3366294.

[12] Sun L., Li S., Li J. and Lv J., "A novel context-based implicit feature extracting method," 2014 International Conference on Data Science and Advanced Analytics (DSAA), Shanghai, 2014, pp. 420-424.

[13] . Singh V. K., Piryani R., Uddin A. and Waila P., "Sentiment analysis of movie reviews: A new feature-based heuristic for aspect-level sentiment classification," 2013 International Mutli-Conference on Automation, Computing, Communication, Control and Compressed Sensing (iMac4s), Kottayam, 2013, pp. 712-717.

[14] Song H., Fan Y., Liu X. and Tao D., "Extracting product features from online reviews for sentimental analysis," 2011 6th International Conference on Computer Sciences and Convergence Information Technology (ICCIT), Seogwipo, 2011, pp. 745-750.

[15] Sun, J., Long, C., Zhu, X., \& Huang, M. (2009). Mining reviews for product comparison and recommendation. Polibits, 39, pp. 33-40

[16] V. Ravi Kumar and Raghuveer K., "Dependency Driven Semantic Approach to Product Features Extraction and Summarization Using Customer Reviews," In Proceedings of the Second International Conference on Advances in Computing and Information Technology (ACITY), Chennai, India - Volume 3, 2012, pp 225-238.

[17] Wu Y., Zhang Q., Huang X., Wu L., "Phrase dependency parsing for opinion mining," Proceedings of the 2009 Conference on Empirical Methods in Natural Language Processing, (Singapore),2009, pp. 1533-1541.

[18] Xu B., Zhao T., Zheng D. and Wang S., "Product features mining based on Conditional Random Fields model," 2010 International Conference on Machine Learning and Cybernetics, Qingdao, 2010, pp. 3353-3357

[19] Yan Z., Xing M., Zhang D., and Ma B., "EXPRS: An extended pagerank method for product feature extraction from online consumer reviews," Information and Management, vol. 52, no. 7. Elsevier B.V., pp. 850-858, 2015.

[20] Zhixing Li, "Product Feature Extraction with a Combined Approach", in Proceedings of the 3rd IEEE International Symposium on Intelligent Information Technology and Security Informatics, pp. 686-690, Jinggangsgan, 2015 